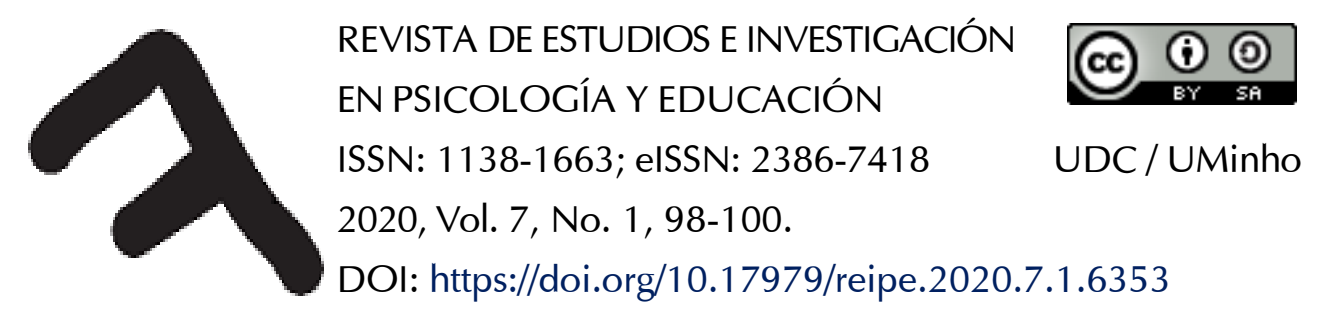

\title{
Reseña del libro: Comunicación simbólica: Comunicación aumentativa y alternativa
}

\begin{abstract}
Review of the book: Symbolic communication: Augmentative and alternative communication
\end{abstract}

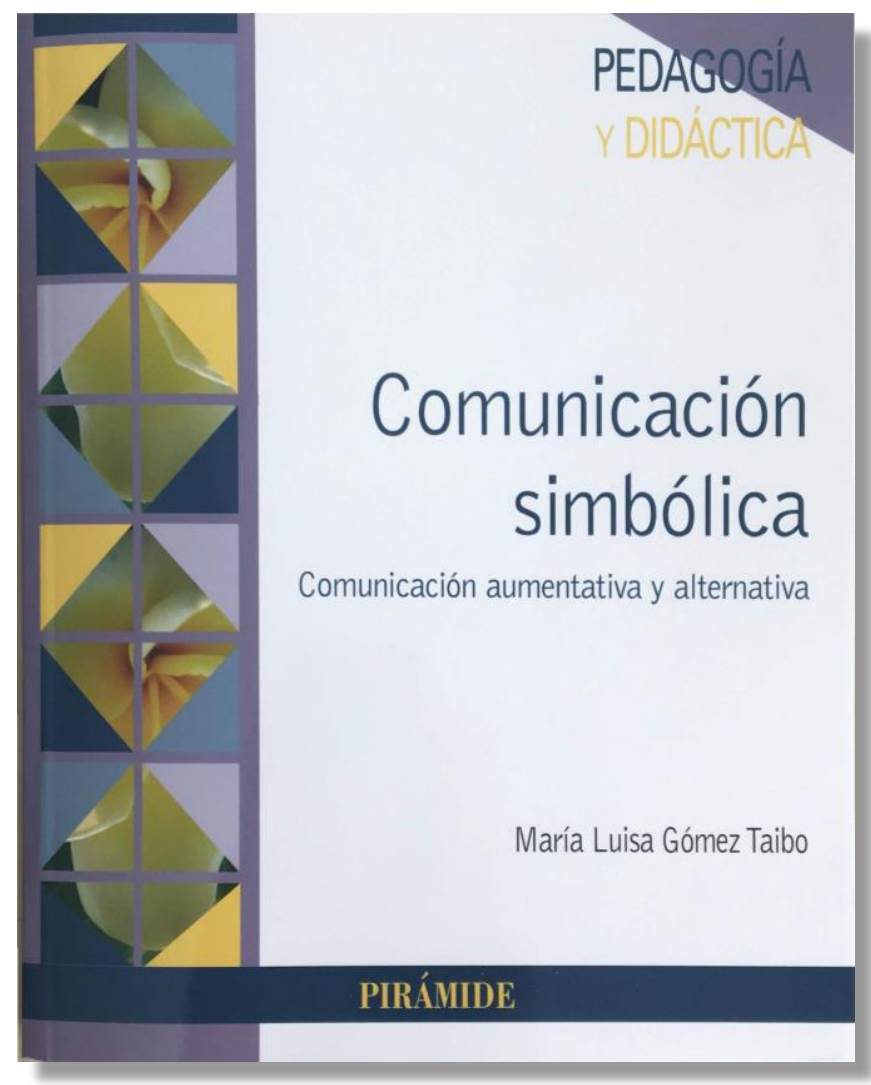

Autora: María Luisa Gómez-Taibo

Departamento de Psicología, Facultad de Ciencias de la Educación, Universidad de A Coruña, España.

Editorial: Pirámide

Lugar de edición: Madrid, España

Número de páginas: 336

Año: 2020

ISBN papel: 978-84-368-4222-7

ISBN ePub.: 978-84-368-4223-4 
Una de las dificultades a las que se enfrentan los profesionales de nuestro país que trabajan en el terreno de la comunicación aumentativa y alternativa (en adelante CAA), es la ausencia de manuales actualizados que reúnan la numerosa pero dispersa literatura existente y la presenten de una forma coherente y accesible. Los manuales existentes (por ejemplo, Sotillo, 2003 o Torres, 2001) tienen ya casi 20 años, y su contenido se hace inevitablemente incompleto, sobre todo en un campo que ha sufrido transformaciones radicales en las dos últimas décadas (McNaughton y Light, 2015). Precisamente por esa necesidad de manuales actualizados, es relevante la publicación de este libro escrito por María Luisa Gómez Taibo, profesora de la Universidade da Coruña, quien ha desarrollado una importante labor docente e investigadora centrada en el estudio y la aplicación de la CAA en personas con discapacidad.

Organizativamente, la obra se articula en torno a siete capítulos. En el Capítulo 1 se abordan las bases conceptuales del tema, empezando por los elementos más básicos como la propia definición de CAA, y terminando por dos aspectos que serán relevantes por cuanto sirven para ordenar el resto del manual: (1) la clasificación del continuo de tipos de comunicación, desde la no simbólica hasta la simbólica, y (2) la distinción entre comunicadores emergentes, principiantes y simbólicos emergentes.

A continuación, el Capítulo 2 está centrado en la valoración de las habilidades comunicativas, un proceso que como la propia autora reconoce, nunca resulta fácil. Por ello se propone ir más allá de las pruebas estandarizadas y utilizar protocolos sensibles con un enfoque ecológico-funcional y dinámico, centrados no sólo en el comunicador sino también en sus compañeros de comunicación y en el entorno.

Los restantes capítulos tratan ya sobre aspectos de intervención, y lo hacen siguiendo tanto el continuo de formas de comunicación como la tipología de comunicadores que se indicaron más arriba. Así, el Capítulo 3 ofrece orientaciones y estrategias para usuarios que no tienen intención comunicativa ni entienden símbolos (comunicadores no simbólicos), y se especifica una secuencia de trabajo para pasar de conductas espontáneas a intencionales, y de conductas intencionales a gestos comunicativos intencionales. El Capítulo 4, por su parte, retoma todas esas estrategias para presentar distintos programas que permitan adquirir la comunicación intencional; específicamente, se describen la promoción para el aprendizaje a través de la interacción activa (o PLAI), la enseñanza naturalista prelingüística y el método de Van Dijk. En el Capítulo 5 se plantea que, una vez conseguida la intencionalidad, estaríamos hablando ya de comunicadores principiantes en los que la intervención debería centrarse en el uso de símbolos de tipo asistido que facilitan la representación de la realidad (por ejemplo, fotografías, pictogramas o símbolos gráficos). Se conseguiría así la emergencia de una comunicación simbólica inicial, que en el Capítulo 6 se extiende para enseñar funciones comunicativas básicas, destacando las relacionadas con la petición y el rechazo y empleando para ello soluciones tanto de baja como de alta tecnología. Por último, en el Capítulo 7 se señala la necesidad de que los comunicadores que ya cuentan con un repertorio mínimo basado en símbolos asistidos, pasen a una inmersión intensiva en un entorno rico lingüísticamente, proponiendo la utilización razonable de las nuevas tecnologías, las técnicas de proyección lingüística rápida y el entrenamiento de matrices como herramientas relevantes.

Haciendo una valoración crítica del contenido de este manual, sin duda el aspecto más importante y cuya inclusión hay que alabar es el papel central que, en todo momento, se otorga a la comunicación simbólica como objetivo fundamental del trabajo de evaluación e intervención. En el terreno actual de la CAA, es fácil distraerse y caer en el error de confundir las herramientas (un sistema alternativo concreto o una aplicación informática específica) con el objetivo (conseguir que se adquiera la comunicación). Afortunadamente, eso no ocurre aquí y a lo largo de los distintos capítulos, todo el peso se pone en lograr la emergencia de la comunicación como fin último de la intervención, independientemente de las herramientas, técnicas y programas que se empleen.

Precisamente por ello, es relevante que el manual no se centre en ningún sistema alternativo concreto, que no se circunscriba a patologías particulares, y que no haga referencia a ningún profesional como responsable de la evaluación y la intervención. De esta manera se evitan algunos de los errores más frecuentes que aquejan a la práctica de la CAA, como son creer que la puesta en marcha de un sistema alternativo es una intervención suficiente para los problemas de las personas con dificultades de comunicación, que las etiquetas diagnósticas 
dicten el tipo de trabajo que debe hacerse, o la imposición de artificiosos límites de competencias entre profesionales en lugar de promover la necesaria colaboración interdisciplinar.

En este mismo sentido, es interesante el papel que se otorga a las nuevas tecnologías que han invadido el campo de la CAA en la forma de dispositivos portátiles y de aplicaciones informáticas. Como no podía ser de otro modo, estas tecnologías aparecen citadas en varios capítulos, pero siempre como herramientas útiles al servicio de un objetivo (la comunicación) y que deben emplearse con precaución, sin caer en la tentación de utilizar la tecnología simplemente porque resulta novedosa o atractiva (McNaughton y Light, 2013).

Por último, también hay que destacar que todos los principios, estrategias y programas que aparecen (tanto de evaluación como de intervención) están actualizados, se basan en buenas prácticas y en las mejores evidencias, y se describen de manera muy detallada, pero al mismo tiempo clara y accesible, facilitando por tanto que puedan ser aplicados por los profesionales en su labor clínica.

Con todo esto, la lectura de este manual es más que recomendable para estudiantes y profesionales cuya labor esté relacionada con la CAA (o vaya a estarlo). Coincido con lo expresado por Francisco Alcantud en el prólogo, cuando señala que "está llamado a ser una referencia obligada en la literatura en castellano sobre los trastornos de la comunicación y el lenguaje y su intervención" (p. 20), pero me permito añadir que lo será no solo porque en nuestro país hiciera falta un manual moderno y actualizado sobre CAA (que también), sino porque lo que hacía falta era, precisamente, este manual.

\section{Referencias}

McNaughton, D. Y Light, J. (2013). The iPad and mobile technology revolution: Benefits and challenges for individuals who require augmentative and alternative communication. Augmentative and Alternative Communication, 29(2), 107-116. https://doi.org/10.3109/07434618.2013.784930

McNaughton, D. y Light, J. (2015). What we write about when we write about AAC: The past 30 years of research and future directions. Augmentative and Alternative Communication, 31(4), 261-270. https://doi.org/10.3109/07434618.2015.1099736

Sotillo, M. (2003). Sistemas alternativos de comunicación. Madrid: Trotta.

Torres, S. (2001). Sistemas alternativos de comunicación. Manual de comunicación aumentativa y alternativa: sistemas y estrategias. Madrid: Ediciones Aljibe.

Francisco Cabello-Luque

Facultad de Psicología

Universidad de Murcia

España

fcabello@um.es

Fecha de recepción: 18 de abril de 2020.

Fecha de aceptación: 25 de abril de 2020.

Fecha de publicación: 1 de julio de 2020. 